\title{
Approximate Solution of Some Classes of Integral Equations Using Bernstein Polynomials of Two-Variables
}

\author{
Haleema S. Ali*
}

Lamyaa H. Ali**

Received 27, October, 2010

Accepted 20, November, 2011

\begin{abstract}
:
The research aims to find approximate solutions for two dimensions Fredholm linear integral equation. Using the two-variables of the Bernstein polynomials we find a solution to the approximate linear integral equation of the type two dimensions. Two examples have been discussed in detail.

Key Word: Bernstein Polynomials, Two-Variables Fredholm linear integral equation
\end{abstract}

\section{Introduction:}

New methods are always needed to solve integral equation because no single method works well for all such equations. There has been considerable interest in solving differential and integral equations using techniques which involve new formal two-variables Bernstein polynomials method.

Now consider the following linear two-dimensional Fredholm integral equations of the second kind [1-3]

$$
\begin{aligned}
& u(x, y)=f(x, y)+\int_{00}^{1} \int_{0}^{1} k(x, y, t, s, u(t, s)) d s d t \\
& \ldots(1) \\
& (x, y) \in D=[0,1] \times[0,1]
\end{aligned}
$$

Where $k: D \times D \times R \rightarrow R$ is a continuous linear given function, $f: D \rightarrow R$ is also continuous given function and the two-variables function $u(x, y)$ is the unknown function.

In this paper we introduce an approximate approach to solve twodimensional linear Fredholm integral equations of the second kind given in (1) using new formal of two-variables Bernstein polynomials method.

\section{Bernstein Polynomials Method with Two-Variables.}

The Bernstein polynomials of twovariables degree of $(n+m)$ can be defined[ 4-6]:

$B_{i j}^{n+m}(x, t)=\left(\begin{array}{l}n \\ i\end{array}\right)\left(\begin{array}{c}m \\ j\end{array}\right) x^{i} t^{j}(1-x)^{n-i}(1-t)^{m-j} \ldots$

where

$\left(\begin{array}{l}n \\ i\end{array}\right)\left(\begin{array}{c}m \\ j\end{array}\right)=\frac{n !}{i !(n-i) !} \frac{m !}{j !(m-j) !}$

and $(n, m)$ are the degree of polynomials and i,j are the index of polynomials

\section{Proposition}

The Bernstein polynomials of degree $\mathrm{n}+\mathrm{m}$ in the terms of the power basis is given by the following formula [ 7]

$$
B_{i j}^{n+m}(x, t)=\sum_{k=i l=j}^{n} \sum^{m}(-1)^{k-i}(-1)^{l-j}\left(\begin{array}{l}
n \\
k
\end{array}\right)\left(\begin{array}{l}
\mathrm{k} \\
\mathrm{i}
\end{array}\right)\left(\begin{array}{l}
\mathrm{m} \\
1
\end{array}\right)\left(\begin{array}{l}
1 \\
\mathrm{j}
\end{array}\right) \mathrm{x}^{\mathrm{k}} t^{l}
$$

2. General Form of the Double integral Using Two- Variables Bernstein Polynomials Method

In this section the Bernstein polynomials of two-variables of degree $(n+m)$ may be used define, the general form, of the double integral as the following lemma

\footnotetext{
*Al- Mustansiriya University/ College of Engineering/ Department of Materials.

**Al- Mustansiriya University/ College of Science/ Department of Mathematics
} 


\section{Lemma}

The double integral in the interval $[0,1] \times[0,1]$, Bernstein polynomials of degree $n, m$ is given by the following formula

$$
\int_{00}^{11} B_{i}^{n+m}(x, t) d x d t=\frac{1}{(n+1)(m+1)} \cdots
$$

\section{Proof}

Directs calculating using the definition of the Bernstein polynomials of two variables and the binomial theorem, as follows

$$
\begin{aligned}
& B_{i j}^{n+m}(x, t)=\left(\begin{array}{l}
n \\
i
\end{array}\right)\left(\begin{array}{l}
\mathrm{m} \\
\mathrm{j}
\end{array}\right) \mathrm{t}^{\mathrm{j}} x^{i}(1-x)^{n-i}(1-t)^{m-j} \\
& =\left(\begin{array}{l}
n \\
i
\end{array}\right)\left(\begin{array}{l}
\mathrm{m} \\
\mathrm{j}
\end{array}\right) \mathrm{t}^{\mathrm{j}} x^{x^{i} \sum_{k=0} \sum_{l=0}-i m-j}(-1)^{k}(-1)^{l}\left(\begin{array}{l}
n-i \\
k
\end{array}\right)\left(\begin{array}{l}
m-j \\
l
\end{array}\right) x^{k} t^{l}
\end{aligned}
$$

Any Bernstein polynomial of degree $n$ can be written in terms of the power

$$
\begin{aligned}
& \text { basis } \quad\left\{1, t, t^{2}, t^{3}, \ldots, t^{n}\right\} \text {, } \\
& \left\{1, x, x^{2}, x^{3}, \ldots, x^{n}\right\} \\
& =\sum_{k=0}^{n-i} \sum_{l=0}^{m-j}(-1)^{k}(-1)^{l}\left(\begin{array}{l}
n \\
i
\end{array}\right)\left(\begin{array}{l}
\mathrm{m} \\
\mathrm{j}
\end{array}\right)\left(\begin{array}{l}
n-i \\
k
\end{array}\right)\left(\begin{array}{l}
m-j \\
l
\end{array}\right) x^{i+k} t^{j+l} \\
& =\sum_{k=0}^{n} \sum_{l=0}^{m}(-1)^{k-i}(-1)^{l-j}\left(\begin{array}{l}
n \\
i
\end{array}\right)\left(\begin{array}{l}
\mathrm{m} \\
\mathrm{j}
\end{array}\right)\left(\begin{array}{l}
n-i \\
k-i
\end{array}\right)\left(\begin{array}{l}
m-j \\
l-j
\end{array}\right) x^{k} t^{l} \\
& B_{i j}^{n+m}(x, t)=\sum_{k=i l=j}^{n} \sum^{m}(-1)^{k-i}(-1)^{l-j}\left(\begin{array}{l}
n \\
k
\end{array}\right)\left(\begin{array}{l}
k \\
i
\end{array}\right)\left(\begin{array}{l}
m \\
l
\end{array}\right)\left(\begin{array}{l}
l \\
j
\end{array}\right) x^{k} t^{l} \\
& \int_{0}^{1} \int_{0}^{1} B_{i j}^{n+m}(x, t) d x d t \\
& =\int_{0}^{1} \int_{0}^{1} \sum_{k=i}^{n} \sum_{l=j}^{m}(-1)^{k-i}(-1)^{l-j}\left(\begin{array}{l}
n \\
k
\end{array}\right)\left(\begin{array}{l}
k \\
i
\end{array}\right)\left(\begin{array}{l}
m \\
l
\end{array}\right)\left(\begin{array}{l}
l \\
j
\end{array}\right) x^{k} t^{l} d x d t \\
& \int_{0}^{1} \int_{0}^{1} B_{i j}^{n+m}(x, t) d x d t= \\
& \int_{0}^{1} \sum_{k=i}^{n}(-1)^{k-i}\left(\begin{array}{l}
n \\
k
\end{array}\right)\left(\begin{array}{l}
k \\
i
\end{array}\right) x^{k} \\
& {\left[\int_{0}^{1} \sum_{l=j}^{m}(-1)^{l-j}\left(\begin{array}{l}
m \\
l
\end{array}\right)\left(\begin{array}{l}
l \\
j
\end{array}\right) t^{l} d t\right] d x}
\end{aligned}
$$

$$
\int_{0}^{1} \int_{0}^{1} B_{i j}^{n+m}(x, t) d x d t=\int_{0}^{1} \sum_{k=i}^{n}(-1)^{k-i}\left(\begin{array}{l}
n \\
k
\end{array}\right)\left(\begin{array}{l}
k \\
i
\end{array}\right) x^{k}\left[\frac{1}{m+1}\right] d x
$$

Therefore,

$$
\begin{gathered}
\int_{0}^{1} \int_{0}^{1} \sum_{k=i l=j}^{n} \sum^{m}(-1)^{k-i}(-1)^{l-j}\left(\begin{array}{l}
n \\
k
\end{array}\right)\left(\begin{array}{l}
k \\
i
\end{array}\right)\left(\begin{array}{l}
m \\
l
\end{array}\right)\left(\begin{array}{l}
l \\
j
\end{array}\right) x^{k} t^{l} d x d t \\
=\frac{1}{(n+1)(m+1)}
\end{gathered}
$$

\section{Solution of Two-Dimensional} Linear Fredholm integral equation with Two-variables Bernstein

\section{Polynomials Method}

In this section, Bernstein polynomials have been used to find the approximate solution for the twodimensional linear Fredholm integral equations, as follows.

Recall the Fredholm integral equation of the second kind given in equation.(1).

$$
u(x, y)=f(x, y)+\iint_{00}^{1} k(x, y, t, s, u(t, s)) d s d t
$$

Let

$$
u(x, y)=\sum_{i=0}^{n} \sum_{j=0}^{m} P_{i j} B_{i j}^{n+m}(x, y)
$$

where

$B_{i j}^{n+m}(x, y)=\sum_{k=i l=j}^{n} \sum_{i}^{m}(-1)^{k-i}(-1)^{l-j}\left(\begin{array}{l}n \\ k\end{array}\right)\left(\begin{array}{l}\mathrm{k} \\ \mathrm{i}\end{array}\right)\left(\begin{array}{l}\mathrm{m} \\ 1\end{array}\right)\left(\begin{array}{l}1 \\ \mathrm{j}\end{array}\right) \mathrm{x}^{\mathrm{k}} y^{l}$

And $P_{i j}$ control points unknown.

Substitution of the relation in equation(5) in equation (1)gives rise to the relation

$$
\begin{aligned}
& \sum_{i=0}^{n} \sum_{j=0}^{m} P_{i j} B_{i j}^{n+m}(x, y)=f(x, y)+ \\
& \int_{0}^{1} \int_{0}^{1} k\left(x, y, t, s, \sum_{i=0}^{n} \sum_{j=0}^{m} P_{i j} B_{i j}^{n+m}(t, s)\right) d s d t \\
& =f(x, y)+\int_{0}^{1} \int_{0}^{1} k\left(\begin{array}{c}
x, y, t, s, \sum_{i=0}^{n} P_{i 1} B_{i 0}^{n+m}(t, s)+\cdots+ \\
P_{i m} B_{i m}^{n+m}(t, s)
\end{array}\right) d s d t
\end{aligned}
$$




$$
=f(x, y)+\int_{0}^{1} \int_{0}^{1} k(x, y, t, s)\left(\begin{array}{l}
P_{00} B_{00}^{n+m}(t, s)+P_{10} B_{10}^{n+m}(t, s)+\cdots \\
+P_{n 0} B_{n 0}^{n+m}(t, s) \\
+P_{01} B_{01}^{n+m}(t, s)+P_{11} B_{11}^{n+m}(t, s)+\cdots \\
+P_{m 1} B_{m 1}^{n+m}(t, s) \\
\cdots+P_{0 m} B_{0 m}^{n+m}(t, s)+P_{1 m} B_{1 m}^{n+m}(t, s)+\cdots \\
P_{n m} B_{n m}^{n+m}(t, s)
\end{array}\right) d s d t
$$

now to find all integration in equation(6).

Then in order to determine $P_{00}, P_{01} \cdots, P_{0 m}, P_{10}, P_{11}, \ldots P_{1 m}, \cdots P_{n m}$, we need $\mathrm{n}$ equations;

Now

Choose $x_{i}, i=1,2,3, \ldots n$ and $\mathrm{y}_{j}, j=1,2,3, \ldots m$ in the interval $[0,1 \times[0,1]$, which give (n) equations. Solve the (n) equations by Gauss elimination to find the values $P_{00}, P_{01} \cdots, P_{0 m}, P_{10}, P_{11}, \ldots P_{1 m}, \cdots P_{n m}$.

\section{Numerical Examples: \\ Example(1)}

Consider the following twodimensional linear Fredholm integral equation of the second kind:

$$
u(x, y)=x+y+2(x+y)\left(1-e^{1}\right)+\int_{0}^{1} \int_{0}^{1}(x+y) e^{s+t} u(s, t) d s d t
$$

with the exact solution $u(x, y)=x+y$

we choose uniform partition with $\mathrm{m}=\mathrm{n}=1,2,3$. Approximated solution for some values of $(\mathrm{x}, \mathrm{y})$ by using twovariables Bernstein polynomials method and exact values $u(x, y)=x+y$.

by using equation(5) let $\mathrm{n}=\mathrm{m}$, for $\mathrm{n}=1$ we get

$$
\begin{aligned}
& u(x, y)=\sum_{i=0}^{1} \sum_{j=0}^{1} p_{i j} B_{i j}^{2} \\
& u(x, y)=\sum_{i=0 j=0}^{1} \sum_{i j}^{1}\left(\begin{array}{l}
1 \\
i
\end{array}\right)\left(\begin{array}{l}
1 \\
j
\end{array}\right) x^{i} y^{j}(1-x)^{1-i}(1-y)^{1-j} \\
& u(x, y)=p_{00} B_{0}^{1}(x) B_{0}^{1}(y)+p_{01} B_{0}^{1}(x) B_{1}^{1}(y) \\
& +p_{10} B_{0}^{1}(y) B_{1}^{1}(x)+p_{11} B_{1}^{1}(x) B_{1}^{1}(y) \\
& u(x, y)=p_{00}(1-x)(1-y)+ \\
& p_{01}(1-x) y+p_{10}(1-y) x+p_{11} x y
\end{aligned}
$$

Substitution of the relation in equation(8) in equation (7)gives rise to the relation

$$
\begin{gathered}
p_{00}(1-x)(1-y)+p_{01}(1-x) y+p_{10}(1-y) x+p_{11} x y \\
=x+y+2(x+y)\left(1-e^{1}\right)+ \\
\int_{0}^{1} \int_{0}^{1}(x+y) e^{s+t}\left[\begin{array}{l}
p_{00}(1-s)(1-t)+p_{01}(1-s) t \\
+p_{10}(1-t) s+p_{11} s t
\end{array}\right] d s d t
\end{gathered}
$$

Where The control points $p_{i j}, \mathrm{i}=0,1$ $\mathrm{j}=0,1$ are found as follows:

Find all integration in equation. Then in order to determine control points $p_{i j}, \mathrm{i}=0,1, \mathrm{j}=0,1 \quad$ we need $\mathrm{n}$ equations; now choose $x_{i}=0,1$ and $y_{j}=0,1$ in the interval $[0,1] x[0,1]$, which gives (n)equations . solve the (n) equations by Gauss elimination to find the values $p_{i j}, \mathrm{i}=0,1, \mathrm{j}=0,1$. we obtain the approximate solution as $u(x, y)=0(1-x)(1-y)+1(1-x) y+1(1-y) x+2 x y$ when $\mathrm{n}=\mathrm{m}=2$ in equation $(5)$

$$
\begin{aligned}
& u(x, y)=\sum_{i=0}^{2} \sum_{j=0}^{2} p_{i j} B_{i j}^{4} \\
& \begin{aligned}
u(x, y) & =\sum_{i=0}^{2} \sum_{j=0}^{2} p_{i j}\left(\begin{array}{c}
2 \\
i
\end{array}\right)\left(\begin{array}{c}
2 \\
j
\end{array}\right) x^{i} y^{j}(1-x)^{2-i}(1-y)^{2-j} \\
u(x, y) & =p_{00} B_{0}^{2}(x) B_{0}^{2}(y)+2 p_{01} B_{0}^{2}(x) B_{1}^{2}(y)+2 p_{10} B_{1}^{2}(x) B_{0}^{2}(y) \\
& +p_{20} B_{2}^{2}(x) B_{0}^{2}(y)+p_{02} B_{0}^{2}(x) B_{2}^{2}(y)+4 p_{11} B_{1}^{2}(x) B_{1}^{2}(y) \\
& +2 p_{21} B_{2}^{2}(x) B_{1}^{2}(y)+2 p_{12} B_{1}^{2}(x) B_{2}^{2}(y)+p_{22} B_{2}^{2}(x) B_{2}^{2}(y)
\end{aligned} \\
& \begin{aligned}
u(x, y) & =p_{00}(1-x)^{2}(1-y)^{2}+2 p_{01} y(1-y)(1-x)^{2}+2 p_{10} x(1-x)(1-y)^{2} \\
& +p_{20} x^{2}(1-y)^{2}+p_{02} y^{2}(1-x)^{2}+4 p_{11} x y(1-x)(1-y) \\
& +2 p_{21} x^{2} y(1-y)+2 p_{12} x(1-x) y^{2}+p_{22} x^{2} y^{2}
\end{aligned}
\end{aligned}
$$

Substitution of the relation in equation(9) in equation (7) gives rise to the relation

$$
\begin{gathered}
p_{00}(1-x)^{2}(1-y)^{2}+2 p_{01} t(1-y)(1-x)^{2}+2 p_{10} x(1-x)(1-y)^{2} \\
+p_{20} x^{2}(1-y)^{2}+p_{02} y^{2}(1-x)^{2}+4 p_{11} x y(1-x)(1-y) \\
+2 p_{21} x^{2} y(1-y)+2 p_{12} x(1-x) y^{2}+p_{22} x^{2} y^{2} \\
=x+y+2(x+y)\left(1-e^{1}\right)+ \\
\int_{0}^{1} \int_{0}^{1}(x+y) e^{s t+}\left[\begin{array}{l}
p_{00}(1-s)^{2}(1-t)^{2}+2 p_{01} t(1-t)(1-s)^{2}+2 p_{10} s(1-s)(1-t)^{2} \\
+p_{20} s^{2}(1-t)^{2}+p_{00} t^{2}(1-s)^{2}+4 p_{11} s t(1-s)(1-t) \\
+2 p_{21} s^{2} t(1-t)+2 p_{12} s(1-s) t^{2}+p_{22} s^{2} t^{2}
\end{array}\right] d s d t
\end{gathered}
$$

Where The control points $p_{i j}, \mathrm{i}=0,1,2$ $\mathrm{j}=0,1,2$ are found as follows:

Find all integration in equation. Then in order to determine control points 
$p_{i j} \quad, \quad \mathrm{i}=0,1,2 \quad \mathrm{j}=0,1,2 \quad$ we need $\mathrm{n}$ equations; now choose $x_{i}=0,1,2$ and $y_{j}=0,1,2$ in the interval $[0,1] x[0,1]$, which gives (n)equations . solve the (n) equations by Gauss elimination to find the values $p_{i j}, \mathrm{i}=0,1,2, \mathrm{j}=0,1,2$. we obtain the approximate solution as $u(x, y)=0 *(1-x)^{2}(1-y)^{2}$

$$
\begin{aligned}
& +2 * 0.5 * y(1-y)(1-x)^{2}+2 * 0.5 * x(1-x)(1-y)^{2} \\
& +1 * x^{2}(1-y)^{2}+1 * y^{2}(1-x)^{2}+4 * 1 * x y(1-x)(1-y) \\
& +2 * 1.5 * x^{2} y(1-y)+2 * 1.5 * x(1-x) y^{2}+2 * x^{2} y^{2}
\end{aligned}
$$

when $n=m=3$ in equation(5) we get

$$
\begin{aligned}
& u(x, y)=\sum_{i=0}^{3} \sum_{j=0}^{3} p_{i j} B_{i j}^{6} \\
& \begin{aligned}
u(x, y) & =\sum_{i=0}^{3} \sum_{j=0}^{3} p_{i j}\left(\begin{array}{l}
3 \\
i
\end{array}\right)\left(\begin{array}{l}
3 \\
j
\end{array}\right) x^{i} y^{j}(1-x)^{3-i}(1-y)^{3-j} \\
u(x, y) & =p_{00} B_{0}^{3}(x) B_{0}^{3}(y)+3 p_{01} B_{0}^{3}(x) B_{1}^{3}(y)+3 p_{02} B_{0}^{3}(x) B_{2}^{3}(y) \\
& +p_{03} B_{0}^{3}(x) B_{3}^{3}(y)+3 p_{10} B_{1}^{3}(x) B_{0}^{3}(y)+9 p_{11} B_{1}^{3}(x) B_{1}^{3}(y) \\
& +9 p_{12} B_{1}^{3}(x) B_{2}^{3}(y)+3 p_{13} B_{1}^{3}(x) B_{3}^{3}(y)+3 p_{20} B_{2}^{3}(x) B_{0}^{3}(y) \\
& +9 p_{21} B_{2}^{3}(x) B_{1}^{3}(y)+9 p_{22} B_{2}^{3}(x) B_{2}^{3}(y)+3 p_{23} B_{2}^{3}(x) B_{3}^{3}(y) \\
& +p_{30} B_{3}^{3}(x) B_{0}^{3}(y)+3 p_{31} B_{3}^{3}(x) B_{1}^{3}(y)+3 p_{32} B_{3}^{3}(x) B_{2}^{3}(y) \\
& +p_{33} B_{3}^{3}(x) B_{3}^{3}(y)
\end{aligned}
\end{aligned}
$$

$u(x, y)=p_{00}(1-x)^{3}(1-y)^{3}+3 p_{01} y(1-y)^{2}(1-x)^{3}+3 p_{02} y^{2}(1-y)(1-x)^{3}$ $+p_{03} y^{3}(1-x)^{3}+3 p_{10} x(1-x)^{2}(1-y)^{3}+9 p_{11} x y(1-x)^{2}(1-y)^{2}$ $+9 p_{12} x y^{2}(1-x)^{2}(1-y)+3 p_{13} x y^{3}(1-x)^{2}+3 p_{20} x^{2}(1-x)(1-y)^{3}$ $+9 p_{21} x^{2} y(1-y)^{2}(1-x)+9 p_{22} x^{2} y^{2}(1-y)(1-x)+3 p_{23} x^{2} y^{3}(1-x)$ $+p_{30} x^{3}(1-y)^{3}+3 p_{31} x^{3} y(1-y)^{2}+3 p_{32} x^{3} y^{2}(1-y)+p_{33} x^{3} y^{3}$
Substitution of the relation in equation(10) in equation (7)gives rise to the relation

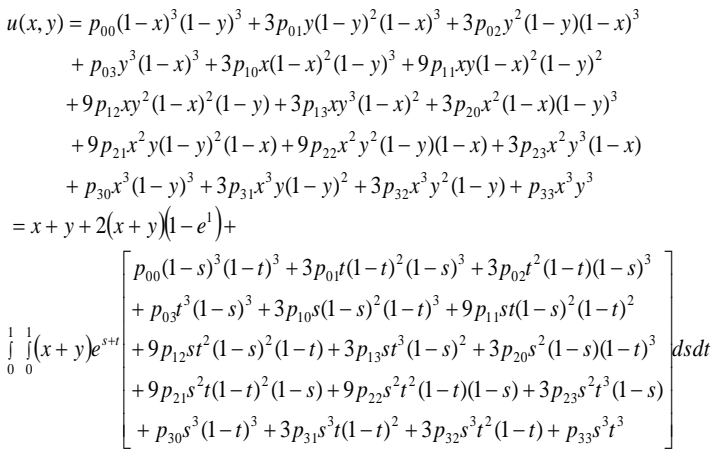

Where The control points $p_{i j}$, $\mathrm{i}=0,1,2,3 \quad \mathrm{j}=0,1,2,3$ are found as follows:

Find all integration in equation above. Then in order to determine control points $p_{i j}, \mathrm{i}=0,1,2,3, \mathrm{j}=0,1,2,3 \quad$ we

\begin{tabular}{|c|c|c|c|c|c|c|c|}
\hline $\mathbf{i}$ & $x$ & $y$ & $\begin{array}{l}\text { Exact } \\
u(x, y)\end{array}$ & $\begin{array}{c}\text { Approximation } \\
u(x, y) n=m=1\end{array}$ & $\begin{array}{c}\text { Approximation } \\
u(x, y) n=m=2\end{array}$ & $\begin{array}{c}\text { Approximation } \\
u(x, y) n=m=3\end{array}$ & error \\
\hline 1 & 0 & 0 & 0 & 0 & 0 & 0 & 0 \\
\hline 2 & $\mathbf{0}$ & 0.25 & 0.2500 & $\mathbf{0 . 2 5 0 0}$ & $\mathbf{0 . 2 5 0 0}$ & 0.2500 & 0 \\
\hline 3 & $\mathbf{0}$ & 0.5 & 0.5000 & 0.5000 & 0.5000 & 0.5000 & 0 \\
\hline 4 & $\mathbf{0}$ & 0.75 & 0.75 & 0.75 & 0.75 & 0.75 & 0 \\
\hline 5 & 0 & 1 & 1 & 1 & 1 & 1 & 0 \\
\hline 6 & 0.25 & 0 & 0.25 & 0.25 & 0.25 & 0.25 & 0 \\
\hline 7 & 0.25 & 0.25 & 0.5 & 0.5 & 0.5 & 0.5 & 0 \\
\hline 8 & 0.25 & 0.5 & 0.75 & 0.75 & 0.75 & 0.75 & 0 \\
\hline 9 & 0.25 & 0.75 & 1 & 1 & 1 & 1 & $\mathbf{0}$ \\
\hline 10 & 0.25 & 1 & 1.25 & 1.25 & 1.25 & 1.25 & 0 \\
\hline 11 & 0.5 & 0 & 0.5 & 0.5 & 0.5 & 0.5 & $\mathbf{0}$ \\
\hline 12 & 0.5 & 0.25 & 0.75 & 0.75 & 0.75 & 0.75 & O \\
\hline 13 & 0.5 & 0.5 & 1 & 1 & 1 & 1 & $\mathbf{0}$ \\
\hline 14 & 0.5 & 0.75 & 1.25 & 1.25 & 1.25 & 1.25 & 0 \\
\hline 15 & 0.5 & 1 & 1.50 & 1.50 & 1.50 & 1.50 & O \\
\hline 16 & 0.75 & 0 & 0.75 & 0.75 & 0.75 & 0.75 & 0 \\
\hline 17 & 0.75 & 0.25 & 0.1875 & 0.1875 & 0.1875 & $\mathbf{0 . 1 8 7 5}$ & $\mathbf{0}$ \\
\hline 18 & 0.75 & 0.5 & 1 & 1 & 1 & 1 & 0 \\
\hline 19 & 0.75 & 0.75 & 1.5 & 1.5 & 1.5 & 1.5 & O \\
\hline 20 & 0.75 & 1 & 1.75 & 1.75 & 1.75 & 1.75 & 0 \\
\hline 21 & 1 & 0 & 1 & 1 & 1 & 1 & 0 \\
\hline 22 & 1 & 0.25 & 1.25 & 1.25 & 1.25 & 1.25 & $\mathbf{0}$ \\
\hline 23 & 1 & 0.5 & 1.5 & 1.5 & 1.5 & 1.5 & 0 \\
\hline 24 & 1 & 0.75 & 1.75 & 1.75 & 1.75 & 1.75 & 0 \\
\hline 25 & 1 & 1 & 2 & 2 & 2 & 2 & O \\
\hline
\end{tabular}
need $\mathrm{n}$ equations; now choose $x_{i}=0,1,2,3$ and $y_{j}=0,1,2,3$ in the interval $[0,1] x[0,1]$, which gives (n)equations . solve the (n) equations by Gauss elimination to find the values $p_{i j}, \mathrm{i}=0,1,2,3, \mathrm{j}=0,1,2,3$.

The results depending on the least square error (L.S.E) is presented in Table(1) .

Table (1) The results of Example(1) 


\section{Example(2)}

Consider the following twodimensional linear Fredholm integral equation of the second kind:

$u(x, y)=x y-\frac{1}{4}(x+y)-\frac{1}{3}+\int_{0}^{1} \int_{0}^{1}(x+y+s+t) u(s, t) d s d t$

with the exact solution $u(x, y)=x y$

we choose uniform partition with $m=n=1,2,3$. Approximated solution for some values of $(\mathrm{x}, \mathrm{y})$ by using twovariables Bernstein polynomials method and exact values $u(x, y)=x y$ when $n=m=3$ in equation (5) we get

$$
\begin{aligned}
& u(x, y)=\sum_{i=0}^{3} \sum_{j=0}^{3} p_{i j} B_{i j}^{6} \\
& u(x, y)=\sum_{i=0}^{3} \sum_{j=0}^{3} p_{i j}\left(\begin{array}{l}
3 \\
i
\end{array}\right)\left(\begin{array}{l}
3 \\
j
\end{array}\right) x^{i} y^{j}(1-x)^{3-i}(1-y)^{3-j} \\
& u(x, y)=p_{00}(1-x)^{3}(1-y)^{3}+3 p_{01} y(1-y)^{2}(1-x)^{3}+3 p_{02} y^{2}(1-y)(1-x)^{3} \\
& +p_{03} y^{3}(1-x)^{3}+3 p_{10} x(1-x)^{2}(1-y)^{3}+9 p_{11} x y(1-x)^{2}(1-y)^{2} \\
& +9 p_{12} x y^{2}(1-x)^{2}(1-y)+3 p_{13} x y^{3}(1-x)^{2}+3 p_{20} x^{2}(1-x)(1-y)^{3} \\
& +9 p_{21} x^{2} y(1-y)^{2}(1-x)+9 p_{22} x^{2} y^{2}(1-y)(1-x)+3 p_{23} x^{2} y^{3}(1-x) \\
& +p_{30} x^{3}(1-y)^{3}+3 p_{31} x^{3} y(1-y)^{2}+3 p_{32} x^{3} y^{2}(1-y)+p_{33} x^{3} y^{3}
\end{aligned}
$$

Substitution of the relation in equation(12) in equation (11)gives rise to the relation

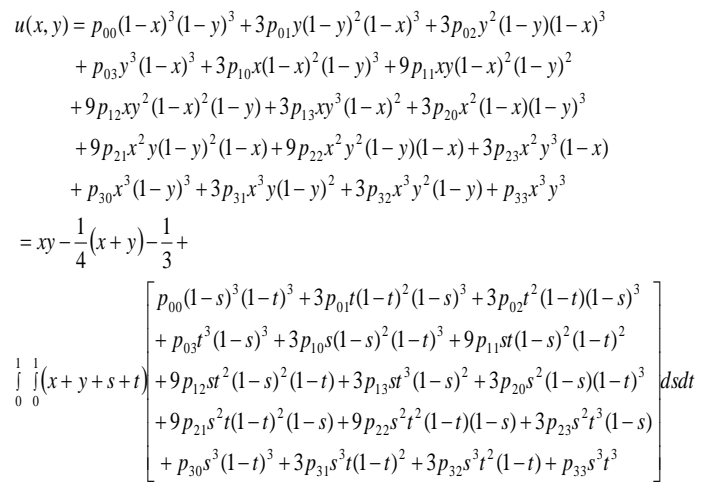

Where The control points $p_{i j}$, $\mathrm{i}=0,1,2,3 \quad \mathrm{j}=0,1,2,3$ are found as follows:

Find all integration in equation. Then in order to determine control points $p_{i j}, \mathrm{i}=0,1,2,3, \mathrm{j}=0,1,2,3$ we need $\mathrm{n}$ equations; now choose $x_{i}=0,1,2,3$ and $y_{j}=0,1,2,3$ in the interval $[0,1] x[0,1]$, which gives (n)equations . solve the (n) equations by Gauss elimination to find the values $p_{i j}, \quad \mathrm{i}=0,1,2,3$, $j=0,1,2,3$. we obtain the approximate

\begin{tabular}{|c|c|c|c|c|c|c|c|}
\hline$i$ & $x$ & $y$ & Exact $u(x, y)$ & Approximation $u(x, y) n=m=1$ & Approximation $(x, y) n=m=2$ & Approximatio $u(x, y) n=m=3$ & Error \\
\hline 1 & $\mathbf{0}$ & 0 & $\mathbf{0}$ & $\mathbf{0}$ & $\mathbf{0}$ & $\mathbf{0}$ & $\mathbf{0}$ \\
\hline 2 & $\mathbf{0}$ & 0.25 & $\mathbf{0}$ & $\mathbf{0}$ & $\mathbf{0}$ & $\mathbf{0}$ & $\mathbf{0}$ \\
\hline 3 & $\mathbf{0}$ & 0.5 & $\mathbf{0}$ & $\mathbf{0}$ & $\mathbf{0}$ & $\mathbf{0}$ & $\mathbf{0}$ \\
\hline 4 & $\mathbf{0}$ & 0.75 & $\mathbf{0}$ & $\mathbf{0}$ & $\mathbf{0}$ & $\mathbf{0}$ & $\mathbf{0}$ \\
\hline 5 & $\mathbf{0}$ & 1 & $\mathbf{0}$ & $\mathbf{0}$ & $\mathbf{0}$ & $\mathbf{0}$ & $\mathbf{0}$ \\
\hline 6 & 0.25 & $\mathbf{0}$ & $\mathbf{0}$ & $\mathbf{0}$ & $\mathbf{0}$ & $\mathbf{0}$ & $\mathbf{0}$ \\
\hline 7 & 0.25 & 0.25 & 0.0625 & 0.0625 & 0.0625 & 0.0625 & $\mathbf{0}$ \\
\hline 8 & 0.25 & 0.5 & 0.1250 & 0.1250 & 0.1250 & 0.1250 & $\mathbf{0}$ \\
\hline 9 & 0.25 & 0.75 & 0.1875 & 0.1875 & 0.1875 & 0.1875 & $\mathbf{0}$ \\
\hline 10 & 0.25 & 1 & 0.25 & 0.25 & 0.25 & 0.25 & $\mathbf{0}$ \\
\hline 11 & 0.5 & $\mathbf{0}$ & $\mathbf{0}$ & $\mathbf{0}$ & $\mathbf{0}$ & $\mathbf{0}$ & $\mathbf{0}$ \\
\hline 12 & 0.5 & 0.25 & 0.1250 & 0.1250 & 0.1250 & 0.1250 & $\mathbf{0}$ \\
\hline 13 & 0.5 & 0.5 & 0.2500 & 0.2500 & 0.2500 & 0.2500 & 0 \\
\hline 14 & 0.5 & 0.75 & 0.3750 & 0.3750 & 0.3750 & 0.3750 & 0 \\
\hline 15 & 0.5 & 1 & 0.50 & 0.50 & 0.50 & 0.50 & 0 \\
\hline 16 & 0.75 & 0 & 0 & 0 & 0 & 0 & 0 \\
\hline 17 & 0.75 & 0.25 & 0.1875 & 0.1875 & 0.1875 & 0.1875 & 0 \\
\hline 18 & 0.75 & 0.5 & 0.3750 & 0.3750 & 0.3750 & 0.3750 & 0 \\
\hline 19 & 0.75 & 0.75 & 0.5625 & 0.5625 & 0.5625 & 0.5625 & 0 \\
\hline 20 & 0.75 & 1 & 0.75 & 0.75 & 0.75 & 0.75 & 0 \\
\hline 21 & 1 & 0 & 0 & 0 & 0 & 0 & 0 \\
\hline 22 & 1 & 0.25 & 0.25 & 0.25 & 0.25 & 0.25 & 0 \\
\hline 23 & 1 & 0.5 & 0.5 & 0.5 & 0.5 & 0.5 & 0 \\
\hline 24 & 1 & 0.75 & 0.75 & 0.75 & 0.75 & 0.75 & 0 \\
\hline 25 & 1 & 1 & 1 & 1 & 1 & 1 & 0 \\
\hline
\end{tabular}
solution as

$$
\begin{aligned}
u(x, y) & =0 *(1-x)^{3}(1-y)^{3}+3 * 0 * y(1-y)^{2}(1-x)^{3}+3 * 0 * y^{2}(1-y)(1-x)^{3} \\
& +0 * y^{3}(1-x)^{3}+3 * 0 * x(1-x)^{2}(1-y)^{3}+9 *\left(\frac{1}{9}\right) * x y(1-x)^{2}(1-y)^{2} \\
& +9 *\left(\frac{2}{9}\right) * x y^{2}(1-x)^{2}(1-y)+3 *\left(\frac{1}{3}\right) * x y^{3}(1-x)^{2}+3 * 0 * x^{2}(1-x)(1-y)^{3} \\
& +9 *\left(\frac{2}{9}\right) * x^{2} y(1-y)^{2}(1-x)+9 *\left(\frac{4}{9}\right) * x^{2} y^{2}(1-y)(1-x)+3 *\left(\frac{2}{3}\right) * x^{2} y^{3}(1-x) \\
& +0 * x^{3}(1-y)^{3}+3 *\left(\frac{1}{3}\right) * x^{3} y(1-y)^{2}+3 *\left(\frac{2}{3}\right) * x^{3} y^{2}(1-y)+1 * x^{3} y^{3}
\end{aligned}
$$

The results depending on the least square error (L.S.E) is presented in Table(2)

Table (2) The results of Example(2) 


\section{Conclusion:}

This paper presents the use of the twovariables Bernstein polynomials method, for solving linear Fredholm two-dimensional integral equation of the second kind. From solving some numerical examples the following points have been identified:

1. This method can be used to solve of linear Fredholm integral equation.

2. It is clear that using the twovariables Bernstein polynomial basis function to approximate when the $\mathrm{n}^{\text {th }}$ degree of Bernstein polynomial increases the error is decreases.

\section{References:}

1. Vasile C. 2001. Numerical Solution of two-dimensional nonlinear Fredholm integral equations of the second kind by spline functions, J. Math .9 (1-2) : 31-48.

2. jerri A. J., 1985. Introduction to Integral Equation with applications,
Marcel Dekker, Inc, New York, First Edition .

3. Lapidus L. and Seinfeid J., 1979. Numerical solution of Differential Equations, Wiley Eastern Limited. New Delhi, Second Edition.

4. Henryk G. and Jose L. P., 2003. On the Approximation Properties of Bernstein polynomials via Probabilistic tools, Boletin de la Asociacion Matematica Venezolana, 1. X(1), 1.

5. Atkinson K. E. ,1997. The Numerical Solution of Integral Equation of the second kind. Cambridge University press, First Edition.

6. Delves L. M. and Mohamed J. L., 1985. Computational Methods for Integral Equations, Cambridge University Press, First Edition .

7.AL-Aisawi H. S., 2006. Computational Methods for Solving Optimal Control Problems, Ph.D. thesis University of Technology.
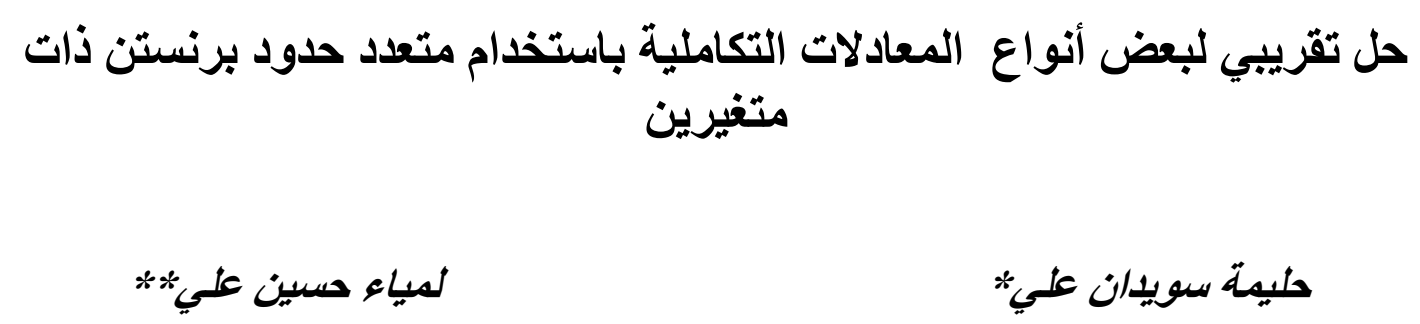

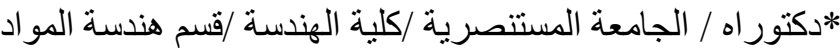

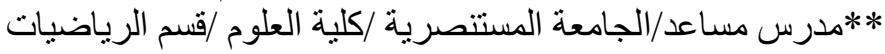

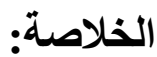

يهذف البحث الى إيجاد الحلول النقريبية لدعادلة فريدهوم الخطية من النوع الثاني ذات البعدين.

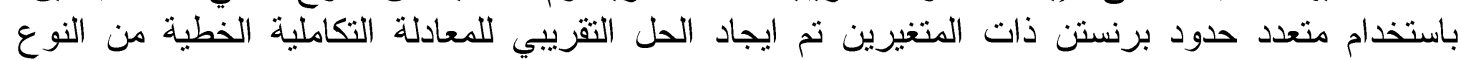
الثاني ذات البعدين. تمت مناقثة مثالين بالتفصيل. 